\title{
The Application and Embodiment of MBE Design Style in the Guidance-Orientated Sign System Design in Zoos
}

\author{
Rui Chen ${ }^{1, *}$
}

${ }^{1}$ Wuhan Textile University, Wuhan, Hubei, China
${ }^{*}$ Corresponding author. Email: 382910331@qq.com

\begin{abstract}
The guidance-orientated sign system is a comprehensive system that effectively conveys relevant information such as humanity and nature to people through the sign medium. It is an effective means to guide people to their destinations efficiently and orderly. Therefore, the design of a systematic and clear guidance-orientated system plays an extremely important role in zoos. With the development of economic culture and the collision of multi-culture, people's aesthetic needs are no longer monotonous. The MBE design style (named after the French designer MBE) has appeared in people's vision and has become the mainstream style of various graphic designs in recent years. MBE's design style is simple, clean, and recognizable to meet people's aesthetic needs. This article takes the application of MBE design style in the guidance-orientated sign system design in zoos as a starting point, and analyzes the characteristics of MBE design style. According to the requirements of the zoos' sign system design, combined with the current status of the zoos' sign system design, finally, the MBE style design rules are applied to the zoos' sign system design, bringing more interesting and personalized visual effects, thereby promoting the overall development of zoos.
\end{abstract}

Keywords: zoos, guidance-orientated sign system, design, MBE style

\section{INTRODUCTION}

With the development of economy and the improvement of people's aesthetic consciousness, the traditional zoo tour instructions are no longer suitable for people's tour experience. The guidance system should follow people's actual needs to adapt to the changes of the times. Zoos have different exhibition areas, and the area there is vast. The reasonable sign system design enables visitors to quickly obtain the information they need. The sign system design not only provides basic guidance functions, but also should be personalized and interesting, reflecting humanistic care and paying attention to the emotional appeal of tourists. This article uses the MBE style to design the guidanceorientated sign system in zoos, which is of great significance to improve the overall image level of the zoo and the tourist experience.

\section{BRIEF DESCRIPTION OF MBE DESIGN} STYLE

\section{A. The concept of $M B E$ design style}

The MBE design style is created by an original designer from Paris, France, and the style name is taken from the designer. This illustration style has gradually evolved from the trend of flat design style and has become one of the popular styles of illustration design in recent years. It is different from the flat style by the wire frame $\mathrm{Q}$ version cartoon style, which is more concise, more focused, and more recognizable.

\section{B. Features of MBE design style}

1) Thick line border and breakpoint: Compared with other styles of illustrations, the most obvious feature of MBE style is the thick line borders, which makes the designer's work more prominent and shining. However, the thick black edges add a sense of depression to the picture and lose its vividness. On this basis, MBE uses a breakpoint method to break the sense of closure of elements, and the staggered lines make the picture flexible and vivid. At the same time, the positions of these breakpoints are arranged according to the shape, and the number of breakpoints is combined with the atmosphere of the picture.

2) The overflow of the shape and color block: It is not only very vivid on the breakpoints and breaklines, but also has a very obvious feature: overflow. The image performance is to highlight the shadow part of the object refracted by the light with the help of the color block overflow in the opposite direction of the highlight. The highlight of the object is formed by the contour offset between the color block and the line, and 
the opposite side is the shadow. This overflow effect makes the elements have more sense of style, be unique and individual, and adds a sense of layering and threedimensionality.

3) Simple and bright color matching: MBE style colors are mainly high-saturation bright colors, and more use contrast colors, monochromatic colors, and similar colors. High-saturation colors give people a bright and warm visual effect, and the unity of color matching appears clearer and more regular. When expressing the number or special parts of objects, it can use colors to distinguish to better convey the content it wants to express. It is not necessary to deliberately maintain the unicity of hue, so that the overall picture is more harmonious and more textured.

4) Unique background decoration elements: In MBE design style icons and illustrations, small dots, small plus signs, and small petals are the most common element graphics. Later, after the evolution of lots of designers, their colors, sizes, and positions are changed, and more types of element graphics are derived, and they are both mellow and regular, simple and interesting.

\section{OVERVIEW OF THE GUIDANCE- ORIENTATED SIGN SYSTEM IN ZOOS}

\section{A. Overview of the current status of the guidance- orientated sign system in zoos}

In the public environment, the sign system plays the role of guidance, explanation, instruction and other functions. It is an important link of the layout of the environment, and also an important part of creating style and improving culture [1].

There is a certain history of foreign sign system research, with many successful cases and experience sharing, and a mature theoretical system has been formed. It is understood that the planning of the sign system of foreign zoos is carried out simultaneously with the park construction plan, making the design style of the entire zoo uniform and clear. Compared with the long-term accumulation of foreign experience, the development time of the Chinese sign system is relatively short, and a sound and mature theoretical system has not been formed yet, and the style positioning suitable for itself has not yet been formed, resulting in the zoo's visual information transmission is not accurate and clear.

\section{B. The function and significance of the guidance- orientated sign system in zoos}

There are two main functions and meanings of the guidance-orientated sign system design in zoos. On the one hand, it serves tourists and provides tourists with concise and easy-to-identify park information, which is convenient for tourists to learn about animal introduction, scenic spot distribution, information consultation, etc., playing the functions of indicating road signs, descriptions, and publicity and warnings [2]. On the other hand, it also serves the park. The guidance-orientated sign system design is an important part of the overall image of the park, and its level is related to the service quality of the park. A good guidance-orientated sign system design can improve the image of the park, facilitate the transmission of culture to tourists, and is more conducive to maintaining the order of the park.

\section{Design requirements for guidance-orientated sign system in zoos}

1) Functionality: The guidance-orientated sign system design in zoos has clear functionality, and mainly plays an important role in identification and instruction. Kevin Lynch believes: "An effective impression is, above all, the object's identifiability, showing itself distinct from other things, and being recognized as an independent entity, this is called recognition [3]. For example, tourists can identify and filter the information according to the layout of the flat area in the park, and then successfully reach the next target location. The guidance-orientated sign system design in zoos should have three functions of: 1 . Providing basic information and orientation guidance services of the park. 2. Introducing animal-related knowledge in the park and improving the knowledge reserve of tourists. 3 . Strengthening the maintenance of infrastructure in the park to achieve the role of publicity and warning.

2) Systematicness: The sign design of the tourism park is systematic, it is the unity of the appearance and connotation of the park, and it is complementary to the principle of integrity [4]. The sign design in the park must be unified with the original landscape. The systematic design and the appearance of the park complement each other, which can not only enhance the tour experience, but also enhance the overall connotation. The sign logo of the zoo not only needs to add personalized design according to the indicators, but also maintain the overall sense of unity and form a complete visual system. For example, the design styles of symbols, patterns, fonts and colors are kept uniform, making the park information clearer.

3) Artistry: The guidance-orientated sign system design in zoos is not only to display information in the park, it is also an important tool for rendering the atmosphere of the park. If there is no artistry, design can only be called manufacturing, not design in the true sense [5]. The design of the sign system should be 
coordinated with the environment of the park, giving people the enjoyment of beauty and art, so that visitors can improve their artistic appreciation while receiving information.

4) Visualization: The visualization of the sign system is to enhance the characteristics of things through refinement and generalization in the natural state of things. For example, the icon design of different types of animal exhibition areas can adopt different and representative animal images, so that visitors can understand the relevant knowledge of animals.

5) Regionalization: In landscape design, humanities and historical factors belong to the spiritual level and are an indispensable and important factor in landscape design [6]. The zoo's sign system should be based on the local area and show visitors the cultural history, customs, and other regional information of the area, so that people can feel the cultural charm there.

\section{Classification of guidance-orientated sign system design in zoos}

1) First-level sign: It is generally set at the entrance of the park and at the intersection of roads. In order to enable visitors to have an overall general understanding of the park, know the location and direction of different exhibition halls, and provide public services, the park will provide transportation location maps and guide maps.

2) Second-level sign: There will be a wide range of secondary signs placed at intersections and along the tour, which mainly include road signs and guide signs indicating areas. The content includes the guide for nearby venues and specific directions for public places. From the content of animal information signs, it can be divided into three types: animal information introduction, safety warning and public public welfare information. 1. Animal information: It is mainly used to introduce the names, habits and characteristics of animals, for tourists to read and understand, and to popularize relevant animal knowledge to tourists and play a role in publicity and education. 2. Safety warning: It provides notifications and warnings on road condition information, fire prevention, electricity protection, and prevention injury to and being injured by animals. It mainly reminds tourists to increase selfprotection awareness and ensure the health and safety of tourists and animals. 3. Public welfare information: It mainly uses signs such as caring for animals, caring for the environment, and prohibiting littering at will to enhance people's awareness of protecting the environment and caring for animals.

\section{THE APPLICATION AND EMBODIMENT OF MBE DESIGN STYLE IN THE GUIDANCE- ORIENTATED SIGN SYSTEM DESIGN IN ZOOS}

\section{A. Design of direction symbols}

Different direction arrows have different ways of using them, and they also have different emotional expressions [7]. For example, the parallel arrows on the upper and lower ends (see "Fig. 1") will make people feel the sense of speed and power. This kind of arrow is mostly used in places where people flow quickly, for example, this kind of arrow is very common in airports, high-speed rail and other places. Arrows that are diagonally cut at the upper and lower ends (see "Fig. 2 ") give people a sense of stability and are mostly used in public places such as schools and shopping malls. The author designed a round arrow based on children's psychological characteristics (see "Fig. 3"), combined with the MBE style, which gives people a kind of visual affinity (see "Fig. 4").

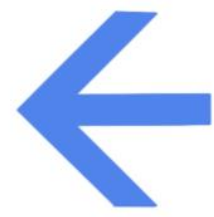

Fig. 1. A parallel arrow on the upper and lower ends.

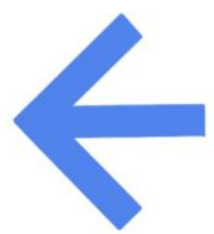

Fig. 2. An arrow that is diagonally cut at the upper and lower ends

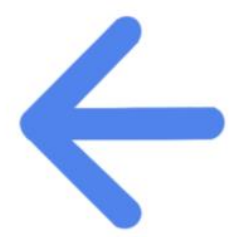

Fig. 3. A round arrow. 


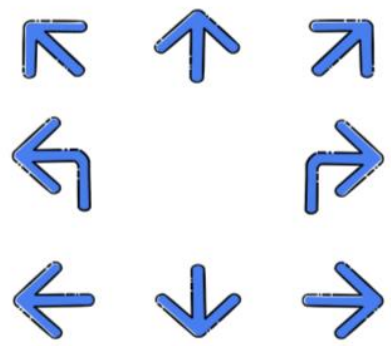

Fig. 4. Arrows of MBE design style.

${ }^{\text {a. }}$ Hand painted by the author.

\section{B. Font design}

Aiming at the font application design in the zoo's sign system, the author applied the MBE design style to the standard Chinese font of "upright, foursquare, coarse, round and simplified style" (see "Fig. 5"), standard English (see "Fig. 6") and numbers (see "Fig. $7 ")$. The fonts with rounded corner and the application of MBE style make the fonts more clear and concise, which is in line with children's psychology.

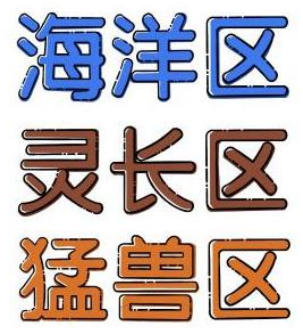

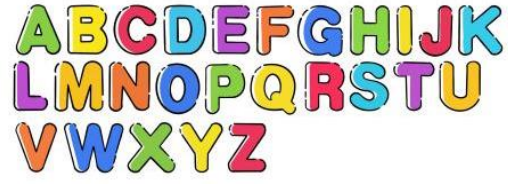

Fig. 6. English font of MBE design style.

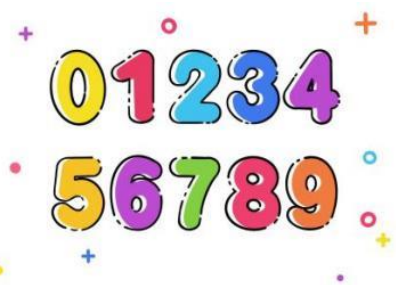

Fig. 7. Numbers of MBE design style.

\section{Animal sign logo design}

This design plan adopts MBE design style combined with the image characteristics of different animals to display the themes of exhibition halls, combining scientificity and enjoyment (see "Fig. 8"). The logo of the exhibition hall is presented in the image of animals, and the information transmission is more accurate, straightforward and vivid.

Fig. 5. Chinese font of MBE design style.

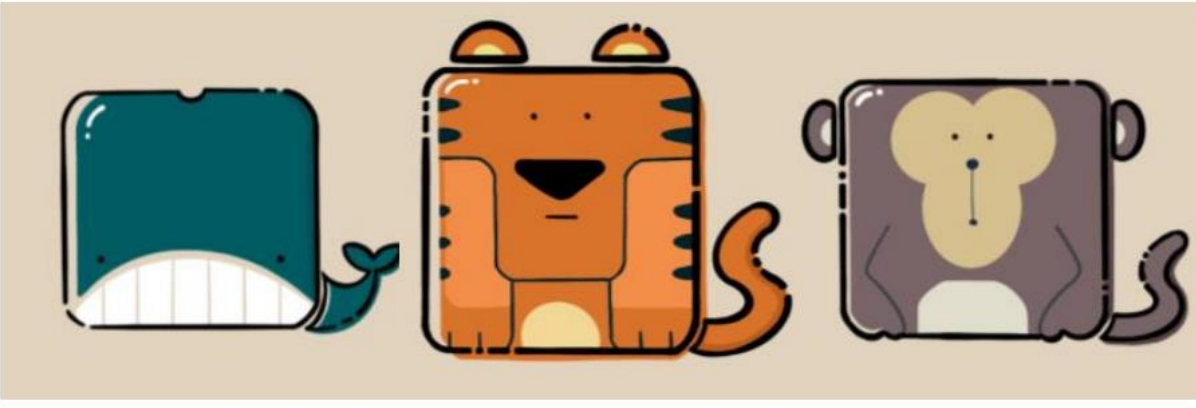

Fig. 8. Animal sign logo design of MBE design style.

D. Safety warning sign design

The design of the safety warning sign should consider the scientificity of the content, but at the same time, it is necessary to add interesting elements to enhance the tourist experience. The MBE design style is adopted to make the sign popular and easy to understand and more accessible (see "Fig. 9"). 


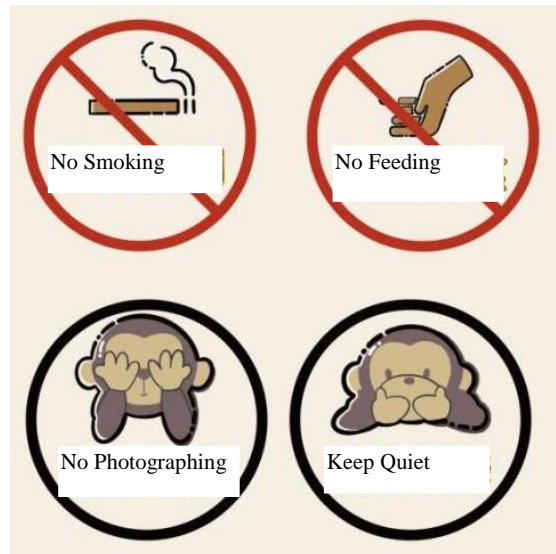

Fig. 9. Safety warning sign design of MBE design style.

a. Hand painted by the author.

\section{CONCLUSION}

With the progress and development of society, more and more fashionable design styles emerge, it's needed to learn to apply it rationally in all areas of life. In this paper, the design and application of MBE style in the sign system of zoos is taken as the starting point, so as to improve the zoos' overall image by improving their guidance-orientated sign design. The characteristics of MBE style are simple, clean, and easy to recognize. While accurately conveying information, it is bound to make visitors experience humanistic care and make them impressed by the park.

\section{References}

[1] Xiao Yong. Sign design [M]. Wuhan: Hubei Fine Arts Publishing House, 2010. (in Chinese)

[2] Zhang Kening. Research on the design of zoos' sign system [J]. Art Science and Technology, 2015.10.15. (in Chinese)

[3] [America] Kevin Lynch. "City image" [M]. Beijing: Huaxia Publishing House, 2001.04. (in Chinese)

[4] Qiu Chen. Research on the design and communication strategy of tourist image signs in cultural scenic spots - taking Xuzhou as an example [J]. Journal of Nanjing University of the Arts, 2014.09.15 (5): 24-25. (in Chinese)

[5] $\mathrm{Xu}$ Ying. Research on the design of zoos' sign system in Wuhan [D]. South-Central University for Nationalities, 2013.04.15. (in Chinese)

[6] Wu Jiahua. Landscape morphology [M]. Beijing: China Building Industry Press, 2010. (in Chinese)

[7] Yu Ming. Research on the optimal design of zoos' sign system in Chongqing $[\mathrm{J}]$. Packaging Engineering, 2015.09.120. (in Chinese) 